\title{
Decidable Compositions of O-minimal Automata ^
}

\author{
Alberto Casagrande ${ }^{1,2,3}$, Pietro Corvaja ${ }^{2}$, Carla Piazza ${ }^{2}$, and Bud Mishra ${ }^{4,5}$ \\ ${ }^{1}$ Istituto di Genomica Applicata, Via J. Linussio, 51, 33100 Udine, Italy \\ 2 DIMI, Università di Udine, Via delle Scienze, 206, 33100 Udine, Italy \\ 3 DISA, Università di Udine, Via delle Scienze, 208, 33100 Udine, Italy \\ ${ }^{4}$ Courant Institute of Mathematical Science, NYU, New York, U.S.A. \\ 5 NYU School of Medicine, 550 First Avenue, New York, 10016 U.S.A.
}

\begin{abstract}
We identify a new class of decidable hybrid automata: namely, parallel compositions of semi-algebraic o-minimal automata. The class we consider is fundamental to hierarchical modeling in many exemplar systems, both natural and engineered. Unfortunately, parallel composition, which is an atomic operator in such constructions, does not preserve the decidability of reachability. Luckily, this paper is able to show that when one focuses on the composition of semi-algebraic o-minimal automata, it is possible to translate the decidability problem into a satisfiability problem over formulæ involving both real and integer variables. While in the general case such formulæ would be undecidable, the particular format of the formulæ obtained in our translation allows combining decidability results stemming from both algebraic number theory and first-order logic over $(\mathbb{R}, 0,1,+, *,<)$ to yield a novel decidability algorithm. From a more general perspective, this paper exposes many new open questions about decidable combinations of real/integer logics.
\end{abstract}

\section{Introduction}

We wish to suggest a novel algebraic framework for the purpose of studying composition of hybrid automata. In this framework, we exploit various algebraic techniques (both semi-algebraic geometric and algebraic-number theoretic) to provide effective procedures to solve reachability problems for at least one important class, namely, semi-algebraic o-minimal hybrid automata. We believe that these techniques are applicable more generally and will motivate further applications to other classes and subclasses of hybrid-automata. Our techniques show how to model state-space evolution (as quantified semialgebraic formulae) separately from the temporal synchronization (modeled as a system of linear algebraic Diophantine equations and inequalities) and yet, seek a combined solution to represent simultaneous arrival at a point in the product state-space by each individual component automaton. In order to obtain this decidability result, we needed to innovate in at least three different

\footnotetext{
^ This work is partially supported by PRIN "BISCA" 2006011235, FIRB "LIBI" RBLA039M7M, two NSF ITR grants, and one NSF EMT grant. Corresponding author: carla.piazza@dimi .uniud.it.
} 
areas: to be precise, (1) theory of automata: how to effectively reduce an automata theoretic problem to an algebraic problem by modeling and by seeking solutions for algebraic systems described via algebraic geometric and number theoretic formulations-thus, circumscribing difficulties faced by the usual finite-quotient-techniques; (2) algorithmic algebra: how to solve a system of equations and inequalities involving semi-algebraic geometric formulae combined with linear algebraic-Diophantine relations-a rather non-trivial problem that had remained unsolved till now, except for the special system arising in case of composition of just two automata (see [1]); and (3) recursive function theory: how to better recognize the boundary separating decidability from undecidability in the context of automata, and along the way, expand the body of techniques applicable to such questions. To the best of our knowledge, this paper is the first to explicitly connect discrete-continuous hybridness of these automata to their algebraic analog of mixed real-integer formulations and also first in proposing how to solve them algorithmically.

The paper is organized as follows: Section 1 and 2 introduce hybrid automata and their parallel composition, respectively; in Section 3, we prove the decidability of linear Diophantine systems with semi-algebraic coefficients and, in Section 4, we show how one can reduce to it the reachability problem for hybrid automata obtained by parallel composition of semi-algebraic o-minimal automata; Section 5 hints some simple applications of the proposed techniques; in Section 6, we discuss some possible extentions and Section 7 summarizes the results presented in the paper and draws some comparisons with related literature. All the missing proofs can be found in [2].

\section{Motivations and Notations}

Since their introduction (see, e.g., [3]), hybrid automata have initiated a new tradition, promising powerful tools for modeling and reasoning about complex engineered or natural systems: e.g., embedded and real time systems, or computational biology, where the resulting analyses are providing many new insights. Unfortunately, in their flexibility in capturing dynamics, resides also their limitations: many different undecidability and complexity results have been proven over general hybrid automata [4] and cast doubt on their suitability as a general tool that can be algorithmized and efficiently implemented. However, if these representations are further restricted, as in the powerful family of o-minimal systems [5], one could hope to still enjoy fidelity of representation that far surpasses that of both discrete models and differential equations, and yet avoid undecidability. In particular, reachability has been shown decidable over semialgebraic o-minimal automata [5].

In order to build a theoretical framework that can also use these hybrid representations in a natural manner, one must shift one's attention to the description of large and complex hybrid systems that can be described in a compositional manner, built out of many elementary modules at many different levels of hierarchy. Since the basic fundamental step in a compositional construction is 
through a parallel composition, an essential desideratum of this new theoretical framework is that the reachability property of the product hybrid automaton be decidable, provided that the component hybrid automata belong to a suitably restricted decidable family of automata, e.g., one in the class of o-minimal automata. In general, the product operation does not assure a closure of decidability property for reachability condition. Nonetheless, in [1], we establish decidability of the reachability condition considering the parallel composition of two semi-algebraic o-minimal automata. Even if such hybrid automata could be used to model interesting systems (e.g., in system biology), the limitation on the number of composable automata poses many restrictions on the applicability of the suggested techniques. To address these shortcomings, in this paper, we have generalized the decidability result for the reachability problem over parallel composition of semi-algebraic o-minimal automata by allowing the composition of an arbitrary number of automata and we extend the applicability of the proposed framework to more complex systems.

\subsection{Basic Notions}

A directed graph is a pair $\langle\mathcal{V}, \varepsilon\rangle$ where $\mathcal{V}$ is a finite set of vertices and $\mathcal{E}$ is a finite set of edges. The functions Source $: \mathcal{E} \longrightarrow \mathcal{V}$ and Dest $: \mathcal{E} \longrightarrow \mathcal{V}$ characterize the vertex exited by an edge and the vertex entered by an edge, respectively. In particular, we say that Source $(e)=v$ and Dest $(e)=v^{\prime}$ are the source and the destination of $e$, respectively. In this paper, when we refer to graphs, we always intend directed graphs. A path ph from $v \in \mathcal{V}$ to $v^{\prime} \in \mathcal{V}$ in $G=\langle\mathcal{V}, \varepsilon\rangle$ is either the vertex $v$, if $v=v^{\prime}$, or a sequence of edges " $e_{1}, \ldots, e_{n}$ " such that, for all $i \in[1, n-1]$, Source $\left(e_{i+1}\right)=\operatorname{Dest}\left(e_{i}\right)$, Source $\left(e_{1}\right)=v$, and Dest $\left(e_{n}\right)=v^{\prime}$. A path $p=" e_{1}, \ldots, e_{n}$ " is a cycle if $e_{1}=e_{n}$ and $n>1$. Moreover, if $e_{i} \neq e_{j}$ for all $i, j \in[1, n-1]$ with $i \neq j$, then we say that $p$ is a simple cycle. The standard definition of cycle requires that the first node coincides with the last one, while in our definition we impose that the first and the last edges are identical. Similarly, the standard definition of simple cycle requires that in the cycle the internal nodes are not repeated, while in our definition we require that the internal edges are not repeated. The two definitions are obviously not equivalent, however, a graph has only a finite number of simple cycles under both definitions. Later on we write $|p|$ to denote the length of the path $p$, i.e., the number of its edges.

Next, we introduce some notations and conventions that we will need to define hybrid automata. Capital letters $X, X^{\prime}, X_{m}$, and $X_{m}{ }^{\prime}$, where $m \in \mathbb{N}$, denote variables ranging over $\mathbb{R}$. Analogously, $Z$ denotes the vector of variables $\left\langle X_{1}, \ldots, X_{d}\right\rangle$ and $Z^{\prime}$ denotes the vector $\left\langle X_{1}{ }^{\prime}, \ldots, X_{d}{ }^{\prime}\right\rangle$. The temporal variables $T$, $T^{\prime}, T_{0}, \ldots, T_{n}$ model time and range over $\mathbb{R}_{\geq 0}$. We use the small letters $p, q, r, s$, ... to denote $d$-dimensional vectors of real numbers. Occasionally, we may use the notation $\varphi\left[X_{1}, \ldots, X_{m}\right]$ to stress the fact that the set of free variables of the first-order formula $\varphi$ is included in the set of variables $\left\{X_{1}, \ldots, X_{m}\right\}$. By extension, if $\left\{Z_{1}, \ldots, Z_{n}\right\}$ is a set of variable vectors, $\varphi\left[Z_{1}, \ldots, Z_{n}\right]$ indicates that the free variables of $\varphi$ are included in the set of components of $Z_{1}, \ldots, Z_{n}$. Moreover, given a formula $\varphi\left[Z_{1}, \ldots, Z_{i}, \ldots, Z_{n}\right]$ and a vector $p$ of the same dimension as 
the variable vector $Z_{i}$, the formula obtained by component-wise substitution of $Z_{i}$ with $p$ is denoted by $\varphi\left[Z_{1}, \ldots, Z_{i-1}, p, Z_{i+1}, \ldots, Z_{n}\right]$. When in $\varphi$ the only free variables are the components of $Z_{i}$, after the substitution we can determine the truth value of $\varphi[p]$.

We are now ready to define hybrid automata. For each node of a graph we have an invariant condition and a dynamic law. The dynamic law may depend on the initial conditions, i.e., on the values of the continuous variables at the beginning of the evolution in the state. The jumps from one discrete state to another are regulated by the activation and reset conditions.

Definition 1 (Hybrid Automata - Syntax). A hybrid automaton $H=\left(Z, Z^{\prime}, V\right.$, $\mathcal{E}, I n v, \mathcal{F}, A c t, R e s)$ of dimension d consists of the following components:

1. $Z=\left\langle X_{1}, \ldots, X_{d}\right\rangle$ and $Z^{\prime}=\left\langle X_{1}{ }^{\prime}, \ldots, X_{d}{ }^{\prime}\right\rangle$ are two vectors of variables ranging over the reals $\mathbb{R}$;

2. $\langle\mathcal{V}, \mathcal{E}\rangle$ is a graph. Each element of $\mathcal{V}$ will be dubbed location.

3. Each vertex $v \in \mathcal{V}$ is labeled by the formula $\operatorname{Inv}(v)[Z]$;

4. $\mathcal{F}$ is a function assigning to each vertex $v \in \mathcal{V}$ a continuous vector field over $\mathbb{R}^{d}$; we will use $f_{v}: \mathbb{R}^{d} \times \mathbb{R}_{\geq 0} \longrightarrow \mathbb{R}^{d}$ to indicate the solution of the vector field $\mathcal{F}(v)$ and $\operatorname{Dyn}(v)\left[Z, Z^{\prime}, T\right] \stackrel{\text { def }}{=} Z^{\prime}=f_{v}(Z, T)$;

5. Each edge $e \in \mathcal{E}$ is labeled by the two formulx $\operatorname{Act}(e)[Z]$ and $\operatorname{Res}(e)\left[Z, Z^{\prime}\right]$;

Note that, without loss of generality, we may consider only hybrid automata whose formulæ are satisfiable. In fact, if this is not the case, we can transform the automaton and eliminate the unsatisfiable formulæ. For instance, if there is an edge $e$ such that $\operatorname{Res}(e)\left[Z, Z^{\prime}\right]$ is unsatisfiable, we can simply delete the edge from the automaton. We use $d(H)$ to denote the dimension of the automaton $H$.

Definition 2 (Hybrid Automata - Semantics). A state $\ell$ of $H$ is a pair $\langle v, r\rangle$, where $v \in V$ is a location and $r=\left\langle r_{1}, \ldots, r_{d}\right\rangle \in \mathbb{R}^{d(H)}$ is an assignment of values for the variables of $Z$. A state $\langle v, r\rangle$ is said to be admissible if $\operatorname{Inv}(v)[r]$ is true.

The continuous reachability transition relation $\stackrel{t}{\rightarrow}$, where $t \geq 0$ is the transition elapsed time, between admissible states is defined as follows:

$\langle v, r\rangle \stackrel{t}{\rightarrow}_{C}\langle v, s\rangle \Longleftrightarrow \begin{aligned} & \left.\text { It holds that } r=f_{v}(r, 0) \text { and it holds that } s=f_{v}(r, t) \text { (see } 1\right), \\ & \text { and for each } t^{\prime} \in[0, t] \text { the formula Inv }(v)\left[f_{v}\left(r, t^{\prime}\right)\right] \text { is true. }\end{aligned}$

The discrete reachability transition relation $\rightarrow_{D}$ between admissible states is defined as follows:

$$
\langle v, r\rangle \stackrel{e}{\rightarrow}_{D}\langle u, s\rangle \Longleftrightarrow \begin{aligned}
& \text { it holds that } e \in \mathcal{E}, \operatorname{Source}(e)=v \text {, Dest }(e)=u \text {, and } \\
& \text { both } \operatorname{Act}(e)[r] \text { and } \operatorname{Res}(e)[r, s] \text { are true. }
\end{aligned}
$$

We write $\ell \rightarrow_{C} \ell^{\prime}$ and $\ell \rightarrow_{D} \ell^{\prime}$ meaning respectively that there exists a $t \in \mathbb{R}_{\geq 0}$ such that $\ell \stackrel{t}{\rightarrow} C \ell^{\prime}$ and that there exists an $e \in \mathcal{E}$ such that $\ell \stackrel{e}{\rightarrow} D \ell^{\prime}$. Moreover, we use the notation $\ell \rightarrow \ell^{\prime}$ to denote that either $\ell \rightarrow_{C} \ell^{\prime}$ or $\ell \rightarrow_{D} \ell^{\prime}$.

Building upon a combination of both continuous and discrete transitions, we can formulate a notion of trace as well as a resulting notion of reachability. A trace is a sequence of continuous and discrete transitions. A point $s$ is reachable from a point $r$ if there is a trace starting from $r$ and ending in $s$. 
Definition 3 (Hybrid Automata - Reachability). Let I be either $\mathbb{N}$ or an initial finite interval of $\mathbb{N}$. A trace of $H$ is a sequence of admissible states $\ell_{0}, \ell_{1}, \ldots, \ell_{i}, \ldots$, with $i \in I$, such that $\ell_{i-1} \rightarrow \ell_{i}$ holds for each $i \in I$ greater than zero; such a trace is also denoted by $\left(\ell_{i}\right)_{i \in I}$.

The automaton $H$ reaches a point $s \in \mathbb{R}^{d}$ (in time $t$ ) from a point $r \in \mathbb{R}^{d}$ if there exists a trace $t r=\ell_{0}, \ldots, \ell_{n}$ of $H$ such that $\ell_{0}=\langle v, r\rangle$ and $\ell_{n}=\langle u, s\rangle$, for some $v, u \in \mathcal{V}$ (and $t$ is the sum of the continuous transitions elapsed times). In such a case, we also say that $s$ is reachable from $r$ in $H$.

Given a hybrid automaton $H$ and trace, $t r$, of $H$, a corresponding path of $t r$ is a path $p h$ obtained by considering the discrete transitions occurring in $t r$. In this case, we also say that $p h$ corresponds to $t r$. Notice that if $t r$ is a trace, then there is a set of corresponding paths of $t r$ and such a set is finite and hence, computable.

We are interested in the reachability problem for hybrid automata, namely, given an automaton $H$, an initial set $I \subseteq \mathbb{R}^{d}$, and a final set $F \subseteq \mathbb{R}^{d}$ we wish to decide whether there exists a point in $I$ from which a point in $F$ is reachable.

An interesting class of hybrid automata is the class of o-minimal automata [5, 6]. The formulæ $\operatorname{Dyn}(v), \operatorname{Inv}(v), \operatorname{Act}(e)$, and Res(e) of such automata are defined in a o-minimal theory for each $v \in \mathcal{V}$ and $e \in \mathcal{E}$. Moreover, their resets are constant, i.e., they do not depend on the point from which the edge is crossed. In the case of o-minimal automata defined by a decidable theory, reachability and temporal logic properties can be decided through bisimulation [5]. O-minimal automata always possess a finite bisimulation quotient whose computation is effective when the o-minimal theory is decidable. An o-minimal and decidable theory is the first-order theory of $(\mathbb{R}, 0,1,+, *,<)[7]$, also known as the theory of semi-algebraic sets.

Definition 4 (Semi-Algebraic O-Minimal Automata). An o-minimal automaton is a hybrid automaton such that Dyn(v), Inv(v), Act(e), and Res(e) are formulx of an o-minimal theory [8] and the truth value of $\operatorname{Res}(e)\left[Z, Z^{\prime}\right]$ does not depend on $Z$, for any $v \in \mathcal{V}$ and $e \in \mathcal{E}$. A semi-algebraic o-minimal automaton is an o-minimal automaton such that Dyn(v), Inv(v), Act(e), and Res(e) are semi-algebraic formulx.

The decidability of reachability problem for such class follows directly from $[5,7]$ and the problem itself has been reduced to the satisfiability of a finite disjunction of formulæ of the form $\operatorname{Reach}(H)(p h)\left[Z, Z^{\prime}, T\right]$ in [9]. In particular, if $H$ is a semi-algebraic o-minimal automaton, then $q \in \mathbb{R}^{d(H)}$ is reachable from $p \in \mathbb{R}^{d(H)}$ in $H$ through a trace whose corresponding path is $p h$ in time $t$ if and only if the formula $\operatorname{Reach}(H)(p h)[p, q, t]$ holds.

\section{Parallel Composition of Hybrid Automata}

Given two or more hybrid automata with distinct variables we are interested in analyzing the reachability problem when we let them run independently.

Definition 5 (Parallel Composition). Let $H_{a}=\left(Z_{a}, Z_{a}{ }^{\prime}, V_{a}, \mathcal{E}_{a}, I_{n} v_{a}, \mathcal{F}_{a}\right.$, Act $_{a}$, Res $\left._{a}\right)$ and $H_{b}=\left(Z_{b}, Z_{b}{ }^{\prime}, V_{b}, \mathcal{E}_{b}, I_{n v_{b}}, \mathcal{F}_{b}, A_{c t}\right.$, Res $\left._{b}\right)$ be two hybrid automata over 
distinct variables. The parallel composition of $H_{a}$ and $H_{b}$ is the hybrid automaton $H_{a} \otimes H_{b}=\left(Z, Z^{\prime}, \mathcal{V}, \mathcal{E}\right.$, Inv, $\mathcal{F}, A c t$, Res $)$, where:

- $Z\left(Z^{\prime}\right)$ is the vector obtained by concatenating $Z_{a}$ and $Z_{b}\left(Z_{a}{ }^{\prime}\right.$ and $Z_{b}{ }^{\prime}$, respectively);

$-\mathcal{V}=\mathcal{V}_{a} \times \mathcal{V}_{b}$

- $\mathcal{E}=\left(\mathcal{E}_{a} \times \mathcal{E}_{b}\right) \cup\left(\mathcal{E}_{a} \times \mathcal{V}_{b}\right) \cup\left(\mathcal{V}_{a} \times \mathcal{E}_{b}\right)$ and $\theta\left(\left\langle e_{a}, e_{b}\right\rangle\right) \stackrel{\text { def }}{=}\left\langle\theta\left(e_{a}\right), \theta\left(e_{b}\right)\right\rangle, \theta\left(\left\langle v_{a}, e_{b}\right\rangle\right) \stackrel{\text { def }}{=}$ $\left\langle v_{a}, \theta\left(e_{b}\right)\right\rangle$, and $\theta\left(\left\langle e_{a}, v_{b}\right\rangle\right) \stackrel{\text { def }}{=}\left\langle\theta\left(e_{a}\right), v_{b}\right\rangle$ for all $\theta \in\{$ Source, Dest $\}$;

- $\operatorname{Inv}\left(\left\langle v_{a}, v_{b}\right\rangle\right)[Z] \stackrel{\text { def }}{=} \operatorname{Inv} v_{a}\left(v_{a}\right)\left[Z_{a}\right] \wedge \operatorname{Inv}_{b}\left(v_{b}\right)\left[Z_{b}\right] ;$

- Dyn $\left(\left\langle v_{a}, v_{b}\right\rangle\right)\left[Z, Z^{\prime}, T\right] \stackrel{\text { def }}{=} D y n_{a}\left(v_{a}\right)\left[Z_{a}, Z_{a}{ }^{\prime}, T\right] \wedge D y n_{b}\left(v_{b}\right)\left[Z_{b}, Z_{b}{ }^{\prime}, T\right] ;$

- $A c t\left(\left\langle e_{a}, e_{b}\right\rangle\right)[Z] \stackrel{\text { def }}{=} \operatorname{Act}_{a}\left(e_{a}\right)\left[Z_{a}\right] \wedge A c t_{b}\left(e_{b}\right)\left[Z_{b}\right], \operatorname{Act}\left(\left\langle e_{a}, v_{b}\right\rangle\right)[Z] \stackrel{\text { def }}{=} A c t_{a}\left(e_{a}\right)\left[Z_{a}\right]$, and $\operatorname{Act}\left(\left\langle v_{a}, e_{b}\right\rangle\right)[Z] \stackrel{\text { def }}{=} A c t_{b}\left(e_{b}\right)\left[Z_{b}\right]$

$-\operatorname{Res}\left(\left\langle e_{a}, e_{b}\right\rangle\right)\left[Z, Z^{\prime}\right] \stackrel{\text { def }}{=} \operatorname{Res}_{a}\left(e_{a}\right)\left[Z_{a}, Z_{a}{ }^{\prime}\right] \wedge \operatorname{Res}_{b}\left(e_{b}\right)\left[Z_{b}, Z_{b}{ }^{\prime}\right], \operatorname{Res}\left(\left\langle e_{a}, v_{b}\right\rangle\right)\left[Z, Z^{\prime}\right] \stackrel{\text { def }}{=}$ $\operatorname{Res}_{a}\left(e_{a}\right)\left[Z_{a}, Z_{a}{ }^{\prime}\right] \wedge Z_{b}{ }^{\prime}=Z_{b^{\prime}}$ and $\operatorname{Res}\left(\left\langle v_{a}, e_{b}\right\rangle\right)\left[Z, Z^{\prime}\right] \stackrel{\text { def }}{=} Z_{a}{ }^{\prime}=Z_{a} \wedge \operatorname{Res}_{b}\left(e_{b}\right)\left[Z_{b^{\prime}} Z_{b}{ }^{\prime}\right]$;

where $v_{a} \in \mathcal{V}_{a}, e_{a} \in \mathcal{E}_{a} v_{b} \in \mathcal{V}_{a}$, and $e_{b} \in \mathcal{E}_{b}$.

Our notion of parallel composition is equivalent to those in $[10,11]$ in the case of disjoint set of events. The discrete graph underlying a parallel composition $H_{a} \otimes H_{b}$ can be a multigraph, i.e., there can be more than one edge connecting two nodes. In particular, this could happen if in either $H_{a}$ or $H_{b}$ there are selfloop edges. Hence, we should introduce labels to distinguish different edges connecting the same pair of nodes. For the sake of simplicity, we avoid this additional labeling in the rest of the paper, when no ambiguity may result.

Example 1. Let us consider the o-minimal automata $H_{a}=\left(\left\langle X_{a}\right\rangle,\left\langle X_{a}{ }^{\prime}\right\rangle, V_{a}, \varepsilon_{a}\right.$,

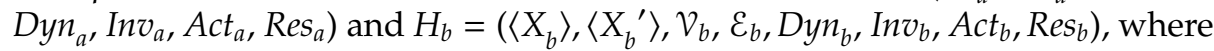
$\mathcal{V}_{i}=\left\{v_{i}\right\}$ and $\mathcal{E}_{i}=\left\{e_{i}\right\}$, for any $i \in\{a, b\}$, and

$$
\begin{array}{rlrl}
H_{a}: & \operatorname{Dyn}_{a}\left(v_{a}\right) \stackrel{\text { def }}{=} \dot{X}_{a}=-1 & H_{b}: \operatorname{Dyn}_{b}\left(v_{b}\right) \stackrel{\text { def }}{=} \dot{X}_{b}=-1 \\
\operatorname{Inv}_{a}\left(v_{a}\right) \stackrel{\text { def }}{=} 0 \leq X_{a} \wedge X_{a} \leq 1 & & \operatorname{Inv}_{b}\left(v_{b}\right) \stackrel{\text { def }}{=} 0 \leq X_{b} \wedge\left(X_{b}\right)^{2} \leq 2 \\
& \operatorname{Act}_{a}\left(e_{a}\right) \stackrel{\text { def }}{=} X_{a}=0 & \operatorname{Act}_{b}\left(e_{b}\right) \stackrel{\text { def }}{=} X_{b}=0 \\
\operatorname{Res}_{a}\left(e_{a}\right) \stackrel{\text { def }}{=} X_{a}{ }^{\prime}=1 & \operatorname{Res}_{b}\left(e_{b}\right) \stackrel{\text { def }}{=}\left(X_{b}{ }^{\prime}\right)^{2}=2 .
\end{array}
$$

The automaton $H_{a} \otimes H_{b}$ is depicted in Figure $1 . H_{a} \otimes H_{b}$ is not o-minimal since it has also identity resets. Moreover, it is possible that a variable is never reset along a cycle of $H_{a} \otimes H_{b}$, e.g., $X_{b}$ is never reset in the cycle " $\left\langle e_{a}, v_{b}\right\rangle,\left\langle e_{a}, v_{b}\right\rangle$ ".

It is easy to prove that $\left(H_{1} \otimes H_{2}\right) \otimes H_{3}$ reaches $q$ from $p$ in time $t$ through a trace tr if and only if $H_{1} \otimes\left(H_{2} \otimes H_{3}\right)$ reaches $q$ from $p$ in time $t$ through $t r$. Hence, we denote by $H_{1} \otimes \ldots \otimes H_{m}$ and $\bigotimes_{i=1}^{m} H_{i}$ the composition of $n$ automata.

As far as reachability is concerned, we first point out that we will study the reachability problem over $\bigotimes_{i=1}^{m} H_{i}$ considering only sets of points of the form $I=\prod_{i=1}^{m} I_{i}$ and $F=\prod_{i=1}^{m} F_{i}$, where $I_{i}, F_{i} \subseteq \mathbb{R}^{d\left(H_{i}\right)}$. To some extent, this simplification will allow us to work on each $H_{i}$ independently. In the general case, our results can be used to both under-estimate and over-estimate reachability. Unfortunately, even with this assumption, one may not always be able 


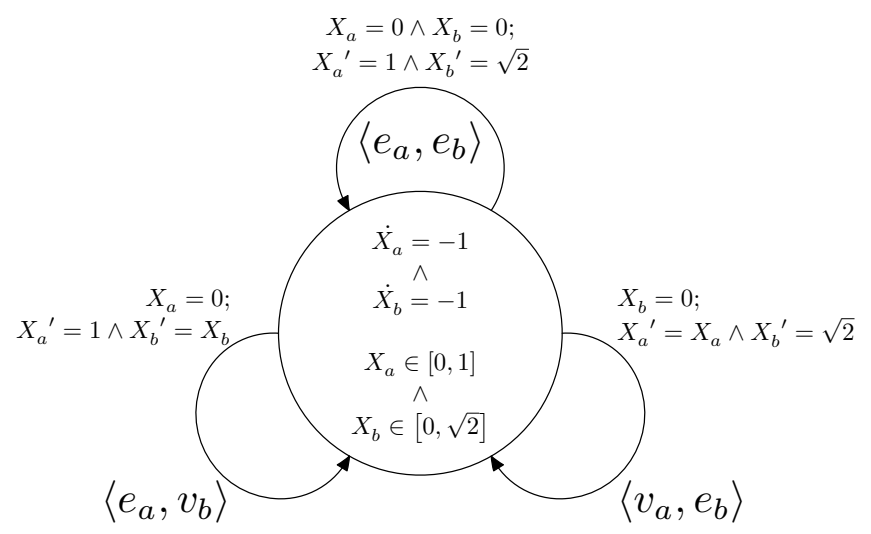

Fig. 1. The hybrid automaton $H_{a} \otimes H_{b}$ of Example 1 .

to ascertain the closure of reachability condition under composition; namely, starting from a set $I_{1}$ it may be possible to reach a set $F_{1}$ in the automaton $H_{1}$ and similarly, starting from a set $I_{2}$ it may be possible to reach a set $F_{2}$ in $H_{2}$, and yet starting from $I_{1} \times I_{2}$ in $H_{1} \otimes H_{2}$ it may not be possible to reach $F_{1} \times F_{2}$. For instance, this happens if $F_{1}$ is reachable only at time $t=1$, while $F_{2}$ is reachable only at time $t=2$. Moreover, the decidability of reachability is not always preserved under parallel composition i.e., it is possible that reachability is decidable over $m$ classes $C_{1}, \ldots, C_{m}$ of hybrid automata, but not over the product class $\bigotimes_{i=1}^{m} C_{i}=\left\{\bigotimes_{i=1}^{m} H_{i} \mid \forall i \in[1, m] H_{i} \in C_{i}\right\}$ (see [11]).

O-minimal hybrid automata have always a finite bisimulation quotient. In [1], we proved that the parallel composition of two o-minimal automata can have an infinite simulation quotient. Hence, the standard quotienting techniques cannot be applied to decide reachability on product automata.

However, it holds that the automaton $\bigotimes_{i=1}^{m} H_{i}$ reaches the set $\prod_{i=1}^{m} F_{i}$ from $\prod_{i=1}^{m} I_{i}$ in time $t$ if and only if $H_{i}$ reaches $F_{i}$ from $I_{i}$ in time $t$ for each $i \in[1, m]$. Hence, in order to study reachability over $\bigotimes_{i=1}^{m} H_{i}$, it would be necessary to better understand the nature of timed reachability over each $H_{i}$ for $i \in[1, m]$.

Definition 6 (Timed-Reachability). Let $H$ be a hybrid automaton. Given $t \geq 0$, $I, F \subseteq \mathbb{R}^{d(H)}$ the $(H, t, I, F)$-timed-reachability problem consists in deciding whether there exist two points $i \in I$ and $f \in F$ such that $H$ reaches $f$ from $i$ in time $t$.

Timed-reachability is in general undecidable. However, the decidability of timed-reachability is preserved by parallel composition and, when $I$ and $F$ are semi-algebraic sets, timed-reachability is decidable over semi-algebraic ominimal automata (see [2]). Unfortunately, decidability of timed-reachability does not imply the decidability of reachability, since there are an infinite number of time instants to be checked.

Intuitively, to decide reachability over the composition of many o-minimal automata, we need to check that we can cycle on their loops elapsing the same 
amount of time. This check involves both integer variables (i.e., the number of times a simple cycle is repeated) and real ones (i.e., the time elapsed on a simple cycle). In the following sections, we first prove a result about decidability of a particular class of Diophantine systems with semi-algebraic coefficients and, then, we reduce the decidability of reachability for parallel composition of an arbitrary number of automata to it.

\section{Linear Systems with Semi-Algebraic Coefficients}

A semi-algebraic set over $\mathbb{R}_{\geq 0}$ is a finite union of intervals and points such that: each interval is characterized by algebraic numbers greater or equal to 0 ; each point is an algebraic number greater or equal to 0 . Semi-algebraic sets are exactly those characterizable through first-order formulæ over $(\mathbb{R}, 0,1,+, *,<)$.

We consider systems of the following form

$$
\left\{\begin{array}{l}
\sum_{i=1}^{n_{1}} A_{i} * \alpha_{i}+\alpha=\sum_{i=1}^{n_{2}} B_{i} * \beta_{i}+\beta \\
\sum_{i=1}^{n_{1}} A_{i} * \alpha_{i}+\alpha=\sum_{i=1}^{n_{3}} C_{i} * \gamma_{i}+\gamma \\
\ldots \\
\sum_{i=1}^{n_{1}} A_{i} * \alpha_{i}+\alpha=\sum_{i=1}^{n_{w}} W_{i} * \omega_{i}+\omega
\end{array}\right.
$$

where capital letters denote variables ranging over $\mathbb{N}_{>0}$, while Greek letters denote real coefficients. In particular, each coefficient can either be a non negative algebraic number or range over a non negative interval characterized by algebraic numbers. Notice that, since the coefficients can range over intervals, this can also be seen as a system of equations and disequations in which some variables range in $\mathbb{N}_{>0}$, while other range in $\mathbb{R}_{\geq 0}$. Intuitively, we can look at it as both a generalization of a linear system of Diophantine disequations and an existential first-order formula involving both integer and real variables. We are interested in the question of satisfiability of such systems.

We distinguish thee cases for the expressions involved in our systems: (a) $\sum_{i=1}^{n_{d}} D_{i} * \delta_{i}+\delta$ is punctual if all the involved coefficients are algebraic numbers; (b) $\sum_{i=1}^{n_{d}} D_{i} * \delta_{i}+\delta$ is quasi-punctual if all $\delta_{i}$ 's are algebraic numbers, while $\delta$ ranges over an interval; (c) $\sum_{i=1}^{n_{d}} D_{i} * \delta_{i}+\delta$ is non-punctual if at least one of the $\delta_{i}$ 's ranges over an interval. An equation is punctual if both its left and right hand sides are punctual. It is quasi-punctual if at least one of the involved expressions is quasi-punctual, while the other one is either punctual or quasi-punctual. It is non-punctual if it involves at least a non-punctual expression.

The algorithm we propose for deciding the satisfiability of System (1) first finds the solutions of the punctual equations. Then these are refined considering the quasi-punctual equations. And in the last step the non-punctual ones come into play. In particular, systems involving only punctual equations can be proved equivalent to linear systems of Diophantine equations, which are decidable [12]. We can deal with the quasi-punctual equations exploiting properties of the additive subgroups of $\mathbb{R}^{q}$ and other results from Diophantine approximations $[13,14]$ : closed subgroups of $\mathbb{R}^{q}$ are decomposable in a discrete component and a dense one; the discrete component requires a "finite" number of checks; 
the dense one is "easy" to deal with. On the one hand, if the punctual together with the quasi-punctual equations admit a finite number of solutions, then we can test them on the non-punctual ones. On the other hand, if the punctual and quasi-punctual equations have an infinite set of solutions, then we can always satisfy also the non-punctual equations. All the details can be found in [2].

Since semi-algebraic sets are composed of a finite number of intervals and points, the techniques presented above not only lead us to the decidability of systems of the form (1) when each coefficient ranges over an interval, but also do so when they range over non negative semi-algebraic sets.

Theorem 1. Let $\mathcal{S}$ be a system of the form (1), where capital letters denote variables ranging over $\mathbb{N}_{>0}$, while Greek letters denote real coefficients ranging over some given semi-algebraic sets included in $\mathbb{R}_{\geq 0}$. The satisfiability of $\mathcal{S}$ is decidable.

\section{Reachability over Parallel Composition}

Now let $H=\bigotimes_{i=1}^{m} H_{i}$ be the parallel composition of $m$ semi-algebraic o-minimal automata. We are interested in the reachability problem over $H$, i.e., we want to check whether the set $F=\prod_{i=1}^{m} F_{i}$ is reachable from $I=\prod_{i=1}^{m} I_{i}$. The considerations presented in Section 2 lead us to the following characterization.

Lemma 1. Let $H_{1}, \ldots, H_{m}$ be $m$ o-minimal hybrid automata. Moreover, for all $i \in$ $[1, m]$, let $I_{i}, F_{i} \subseteq \mathbb{R}^{d\left(H_{i}\right)}$ be sets of points characterized by the first-order formulx $\mathcal{I}_{i}\left[Z_{i}\right]$ and $\mathcal{F}_{i}\left[Z_{i}\right]$, respectively. It holds that $\bigotimes_{i=1}^{m} H_{i}$ can reach $\prod_{i=1}^{m} F_{i}$ from $\prod_{i=1}^{m} I_{i}$ if and only if, for all $i \in[1, m]$, there is a path $p h_{i}$ in $H_{i}$ such that the following formula holds ${ }^{6}$.

$$
\exists T \geq 0 \exists Z_{1}, \ldots, Z_{m} \exists Z_{1}{ }^{\prime}, \ldots, Z_{m}{ }^{\prime} \bigwedge_{i=1}^{m}\left(\mathcal{I}_{i}\left[Z_{i}\right] \wedge \operatorname{Reach}\left(H_{i}\right)\left(p h_{i}\right)\left[Z_{i}, Z_{i}{ }^{\prime}, T\right] \wedge \mathcal{F}_{i}\left[Z_{i}{ }^{\prime}\right]\right)
$$

Unfortunately, the model suggested by above lemma does not immediately provide any decidability result, since we have to consider also an infinite number of cyclic paths. In fact, it may be the case that in order to synchronize all the automata, it is necessary to spend some time over their cycles.

To construct a decidable characterization for reachability over parallel compositions, we exploit the existence of a canonical path decomposition: namely, given a semi-algebraic o-minimal hybrid automaton, from any cyclic path of the automaton, we can extract both an acyclic part, by removing all the cycles occurring in it, and a set of simple cycles. The global time necessary to cover the path is then equal to the sum of the time necessary to cover the acyclic part plus multiples of the times we can spend over the simple cycles. What is important is that in the case of o-minimal automata the time we can spend over a cycle does not depend on the starting and ending point. We define the operation which allows us to add a simple cycle to a path.

\footnotetext{
${ }^{6}$ The formula $\operatorname{Reach}(H)(p h)\left[Z, Z^{\prime}, T\right]$ has been defined in [15].
} 
Definition 7 (Path Augmentability). Let $p h, p h^{\prime}$ be two paths. We say that $p h^{\prime}$ is augmentable to $p h$ if $\mathrm{ph}^{\prime}$ is a simple cycle starting and ending with the edge e and $p h$ is a path involving the edge e. If $p h^{\prime}$ is augmentable to $p h$ we denote by $p h \oplus p h^{\prime}$ the path obtained by inserting $p h^{\prime}$ in ph over the first occurrence of their common edge e, i.e., if $p h^{\prime}=" e, p h_{1}^{\prime}, e$ " and $p h=" e_{1}, \ldots, e_{i-1}, e, e_{i+1} \ldots, e_{n}$ " where we explicitly identify the first occurrence of $e$, then $p h \oplus p h^{\prime}=" e_{1}, \ldots, e_{i-1}, e, p h_{1}^{\prime}, e, e_{i+1} \ldots, e_{n} "$

Let $\mathrm{PH}^{\prime}$ be a set of (simple cyclic) paths we say that $\mathrm{PH}^{\prime}$ is augmentable to a path ph if either $\mathrm{PH}^{\prime}=\emptyset$ or there exists an ordering $p h_{1}, \ldots, p h_{l}$ of the elements of $\mathrm{PH}^{\prime}$ such that for each $i \in[1, l]$ either $p h_{i}$ is augmentable to ph or there exists $j<i$ such that $p h_{i}$ is augmentable to $p h_{j}$.

Notice that if $p h^{\prime}$ is augmentable to $p h$, then it is augmentable to $p h \oplus p h^{\prime}$ also. Moreover, if $p h$ is a cyclic path, then there exist $p h_{1}, \ldots, p h_{n}$, simple cyclic and acyclic, such that $p h=p h_{1} \oplus \ldots \oplus p h_{n}$.

Let $H$ be an o-minimal hybrid automaton and let $p h=" e_{1}, \ldots, e_{m}$ " be a path of $H$. We define the following formula

$$
\begin{array}{r}
\widetilde{\operatorname{Reach}}(H)(p h)\left[Z, Z^{\prime}, T\right] \stackrel{\text { def }}{=} \exists \bar{Z}, \overline{Z^{\prime}}\left(\overline{\operatorname{Reach}}(H)\left(e_{1}\right)[Z, \bar{Z}] \wedge \overline{\operatorname{Reach}}(H)\left(e_{m}\right)\left[\overline{Z^{\prime}}, Z^{\prime}\right] \wedge\right. \\
\left.\operatorname{Reach}(H)\left({ }^{\prime \prime} e_{2}, \ldots, e_{m-1}{ }^{\prime \prime}\right)\left[\bar{Z}, \overline{Z^{\prime}}, T\right]\right)
\end{array}
$$

where $\overline{\operatorname{Reach}}(H)(e)\left[Z, Z^{\prime}\right] \stackrel{\text { def }}{=}\left(\operatorname{Act}(e)[Z] \wedge \operatorname{Res}(e)\left[Z, Z^{\prime}\right]\right)$. It is easy to see that the above formula characterizes all the traces, corresponding to $p h$, which start and end with a discrete transition. Because of the constant reset condition imposed on o-minimal automata, if both the formulæ $\widetilde{\operatorname{Reach}}(H)(p h)[a, b, t]$ and $\widetilde{\text { Reach }}(H)(p h)\left[c, d, t^{\prime}\right]$ hold, then $\widetilde{\text { Reach }}(H)(p h)\left[a, b, t^{\prime}\right]$ holds also.

It follows that, if $H$ is an o-minimal automaton, then we can use the formula Reach $(H)(p h)\left[Z, Z^{\prime}, T\right]$ to define the set of time instants Time $(p h)$ in which $p h$ can be covered, i.e., Time $(p h) \stackrel{\text { def }}{=}\left\{t \mid \exists Z, Z^{\prime} \widetilde{R e a c h}(H)(p h)\left[Z, Z^{\prime}, t\right]\right.$ holds $\}$. Notice that, since $H$ is o-minimal by hypothesis, for each path $p h$ of $H$ the set Time(ph) is o-minimal. It is easy to see that if a path $p h^{\prime}$ is augmentable to a path $p h$ and $t$ is the time needed to evolve through $p h$ then the automaton can elapse a time $t+t^{\prime}$, where $t^{\prime} \in \operatorname{Time}\left(p h^{\prime}\right)$, to evolve through $p h \oplus p h^{\prime}$.

By using observations such as these, we can deduce the following lemma, which characterizes the existence of a trace with elapsed time $t$, without having to examine an infinite number of formulæ.

Lemma 2. Let $H$ be an o-minimal hybrid automaton, let $r, s \in \mathbb{R}^{d(H)}$ and let $t \in \mathbb{R}_{\geq 0}$. There exists a path ph such that Reach $(H)(p h)[r, s, t]$ holds if and only if there exist a path $p h_{0}$ and a set of paths $\mathrm{PH}$ such that: (1) $p h_{0}$ is acyclic; (2) $\mathrm{PH}=\left\{p h_{1}, \ldots p h_{l}\right\}$ is augmentable to $p h_{0}$; (3) we can choose $\alpha$, a vector $\left\langle A_{1}, \ldots, A_{n}\right\rangle \in \mathbb{N}_{>0}^{n}$ and a vector $\left\langle\alpha_{1}, \ldots, \alpha_{n}\right\rangle \in \mathbb{R}_{\geq 0}^{n}$, with $\left\{\alpha_{k_{j}}, \ldots, \alpha_{\left(k_{(j+1)}-1\right)}\right\} \in \operatorname{Time}\left(p h_{j}\right)$ and $1=k_{1}<\ldots<k_{l+1}=$ $n+1$, such that Reach $(H)\left(p h_{0}\right)[r, s, \alpha]$ holds and $t=\alpha+\sum_{i=1}^{n} A_{i} * \alpha_{i}$.

This result suggests a class of verification techniques for timed-reachability on o-minimal automata, but avoids testing an infinite set of formulæ. Moreover, exploiting such result, we can propose the following characterization. 
Theorem 2. Let $H_{1}, \ldots, H_{m}$ be o-minimal automata and $I_{j}, F_{j} \subseteq \mathbb{R}^{d\left(H_{j}\right)}$ be characterized by the first-order semi-algebraic formulx $\mathcal{I}_{j}\left[Z_{j}\right], \mathcal{F}_{j}\left[Z_{j}\right]$ for all $j \in[1, m]$. The automaton $\bigotimes_{i=1}^{m} H_{i}$ reaches $\prod_{i=1}^{m} F_{i}$ from $\prod_{i=1}^{m} I_{i}$ if and only if, for each $h \in\{1, \ldots, m\}$, there exist an acyclic path $p h_{h}$, a set of paths $\mathrm{PH}_{h}=\left\{p h_{h, 1}, \ldots, p h_{h, m_{h}}\right\}$, augmentable to $p h_{h}$, a vector $\left\langle A_{h, 1}, \ldots, A_{h, m_{h}}\right\rangle \in \mathbb{N}_{>0}^{m_{h}}$, and a vector $\left\langle\alpha_{h, 1}, \ldots, \alpha_{h, m_{h}}\right\rangle \in \mathbb{R}_{\geq 0}^{m_{h}}$ such that $\left\{\alpha_{k_{h, j}}, \ldots, \alpha_{\left(k_{h, j+1}-1\right)}\right\} \in \operatorname{Time}\left(p h_{h, j}\right)$, with $1=k_{h, 1}<\ldots<k_{h, m_{h}+1}=m_{h}+1$, and there is $\alpha_{h} \in \mathbb{R}_{\geq 0}$, satisfying both $\exists Z_{h^{\prime}} Z_{h}{ }^{\prime}\left(\operatorname{Reach}\left(H_{h}\right)\left(p h_{h}\right)\left[Z_{h^{\prime}} Z_{h}{ }^{\prime}{ }^{\prime}, \alpha_{h}\right] \wedge \mathcal{I}_{h}\left[Z_{h}\right] \wedge \mathcal{F}_{h}\left[Z_{h}{ }^{\prime}\right]\right)$ and the system

$$
\left\{\begin{array}{l}
\sum_{i=1}^{n_{1}} A_{1, i} * \alpha_{1, i}+\alpha_{1}=\sum_{i=1}^{n_{2}} A_{2, i} * \alpha_{2, i}+\alpha_{2} \\
\sum_{i=1}^{n_{1}} A_{1, i} * \alpha_{1, i}+\alpha_{1}=\sum_{i=1}^{n_{3}} A_{3, i} * \alpha_{3, i}+\alpha_{3} \\
\cdots \\
\sum_{i=1}^{n_{1}} A_{1, i} * \alpha_{1, i}+\alpha_{1}=\sum_{i=1}^{n_{m}} A_{m, i} * \alpha_{m, i}+\alpha_{m}
\end{array}\right.
$$

The number of both acyclic and simple cyclic paths of a hybrid automaton can be bounded from above. Moreover, given a semi-algebraic set $S \subseteq \mathbb{R}$, we can compute the number of its connected components. Since, by Theorem 1, we can decide systems such as the one shown above (Eq. 2), we get the following result.

Corollary 1. Let $H_{1}, \ldots, H_{m}$ be semi-algebraic o-minimal hybrid automata. For all $j \in[1, m]$, let $I_{j}, F_{j} \subseteq \mathbb{R}^{d\left(H_{j}\right)}$ be sets of points characterized by first-order semi-algebraic formulx. Whether $\bigotimes_{j=1}^{m} H_{j}$ reaches $\prod_{j=1}^{m} F_{j}$ from $\prod_{j=1}^{m} I_{j}$ is decidable.

In this direct formulation of the positive result stating the decidability of reachability problem, we have simply focused on the existence of a decision procedure and not its time or space complexity. Furthermore, the infiniteness of simulation quotient gives a hint of its inherent "hardness". However, since the problem is central to any program that focuses on a modular and hierarchical representation of hybrid automata, further work will need to be devoted to the complexity issues. From what we wrote in Section 3, we can deduce an algorithm which, in some (but frequent) cases, decides the reachability problem over parallel composition of o-minimal hybrid automata with a small overhead with respect to the time needed to decide the reachability problem over its components.

Corollary 2. Let $H_{1}, \ldots, H_{m}$ be semi-algebraic o-minimal hybrid automata. For all $j \in[1, m]$, let $I_{j}, F_{j} \subseteq \mathbb{R}^{d\left(H_{j}\right)}$ be sets of points characterized by first-order semi-algebraic formulx. If, for all $j \in[1, m]$, there exists an acyclic path $p^{\prime}{ }^{\prime}$, a cyclic path ph', and a proper interval $O_{j} \subseteq \mathbb{R}_{\geq 0}$ such that $p h_{j}^{\prime \prime}$ is augmentable to $p h_{j}^{\prime}, O_{j} \subseteq$ Time $\left(p h_{j}^{\prime \prime}\right)$, and $H_{j}$ reaches $F_{j}$ from $I_{j}$ through $p h_{j}^{\prime}$, then $\bigotimes_{j=1}^{m} H_{j}$ reaches $\prod_{j=1}^{m} F_{j}$ from $\prod_{j=1}^{m} I_{j}$.

Hence, if the hypothesis in the above corollary holds, the reachability problem is compositional and can be decided by testing each component separately. In this case on each component we can apply either the bisimulation based algorithm proposed in [5] or the semi-algebraic geometry based one proposed in [9].

\section{Applications in System Biology}

As a first example assume that we are monitoring a patient who is under therapy with two drugs, $X$ and $Y . X$ and $Y$ have non-commensurate degradation curves 
and, hence, they cannot be always injected at the same time. Let $X^{\prime}=f_{x}(X, T)$ and $Y^{\prime}=f_{y}(Y, T)$ be degradation curves of $X$ and $Y$. We can imagine that the levels of $X$ and $Y$ have to stay in the ranges $\left[x_{m}, x_{M}\right]$ and $\left[y_{m}, y_{M}\right]$, respectively. When the machine monitoring the patient found that $X$ is in the critical range $\left[x_{m}, x_{l}\right]$ (near the lowest admissible value) it injects $X$. Similarly, when $Y$ enters in the critical range $\left[y_{m}, y_{l}\right]$ it is injected. We can model this situation with the hybrid automata depicted in Figure 2. Since there are some interactions between
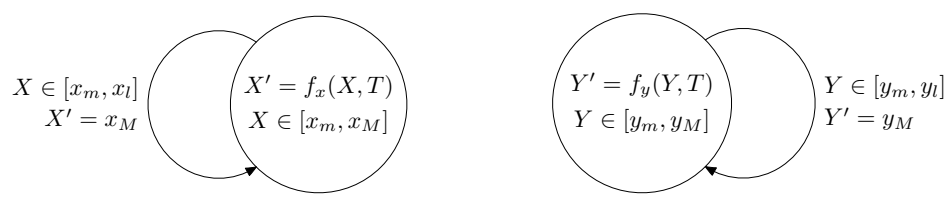

Fig. 2. The hybrid automata depicting a clinical application of parallel composition.

$X$ and $Y$, we can imagine that, if $X$ is in the interval $\left[x_{a}, x_{b}\right]$ and at the same time $Y$ is in the interval $\left[y_{a}, y_{b}\right]$, the patient can have some problems. Let us assume that at time $t=0$ the drug levels are $x_{0}$ and $y_{0}$, respectively. We have to check if in the product of these automata the region $\left[x_{a}, x_{b}\right] \times\left[y_{a}, y_{b}\right]$ is reachable from the initial point $\left\langle x_{0}, y_{0}\right\rangle$. This check can be performed automatically. If the answer is positive, then critical ranges have to be refined.

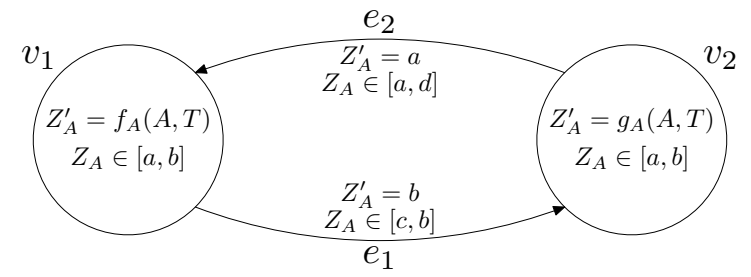

Fig. 3. The hybrid automaton representing the expression level of gene $A$.

Our second example concerns the analysis of metabolic pathways. Imagine we are studying two genes $A$ and $B$ involved in the same pathway. We have some time evolution traces for the expression levels of both $A$ and $B$. Analyzing the traces of $A$ we observe that we can represent its behavior with the automaton depicted in Figure 3. Similarly, we can draw an analogous automaton for $B$. We can now exploit these automata to study whether there exists a strong interaction between $A$ and $B$. For instance if $A$ is a repressor for $B$ and vice-versa, then in the product of the two automata from a region near the maximum value of $A$ and the minimum of $B$ it should be possible to reach a region near the minimum of $A$ and the maximum of $B$ and vice-versa. 
In [16] we proposed a method to translate sets of gene profiles data coming from Real-Time PCR experiments into semi-algebraic hybrid automata. In particular, we proposed clustering techniques which allow to reduce the dimensions of the involved automata. The automata we used in [16] are not o-minimal, since some edges can involve reset conditions of the form $\bigvee_{i=1}^{r}\left(Z=a_{i} \wedge Z^{\prime}=b_{i}\right)$. However, since the disjunctions range over finite sets, it is immediate to translate them into o-minimal semi-algebraic automata with multiple edges. Hence, the reasoning proposed above on genes $A$ and $B$ can be applied and generalized in that context, in order to infer relationships between genes. The combination of the techniques proposed in [16] with the results of this paper suggests us to build one hybrid automaton for each cluster of genes and then to use their parallel composition for the analysis of the relationships between different clusters. The fact of building one hybrid automaton for each cluster ensures us to get substantial reductions on the automaton dimensions, as proved in [16]. The results presented in this paper allow us to combine and compare the behaviors of different clusters represented by separate automata.

\section{Synchronizing Automata and Exchanging Information}

As noticed above, parallel composition provides a powerful and theoretically clean way of modeling complex systems by combining simple component models. However, since the original hybrid automata should not share variables by definition, components cannot "communicate" in models built by parallel composition, i.e., they evolve in isolated environments without interacting. Still, the results of Section 4 can be used to prove the decidability of reachability problem over a different kind of composition operator, similar to that proposed in [10, 11], which allows both interactions and synchronizations between components during system evolution. For the sake of example, let us consider the two semialgebraic o-minimal hybrid automata $H_{1}=\left(Z_{1}, Z_{1}^{\prime}, V_{1}, \mathcal{E}_{1}, I n v_{1}, \mathcal{F}_{1}, A c t_{1}\right.$, Res $\left._{1}\right)$ and $H_{2}=\left(Z_{2}, Z_{2}{ }^{\prime}, V_{2}, \mathcal{E}_{2}, I n v_{2}, \mathcal{F}_{2}, A_{c t_{2}}\right.$, Res $\left._{2}\right)$ depicted in Figure 4, where:

$$
\begin{array}{rr}
H_{1}: Z_{1}=\left\langle X_{1}, X_{2}, X_{3}\right\rangle & H_{2}: Z_{2}=\left\langle X_{2}\right\rangle \\
& \operatorname{Dyn}_{1}\left(v_{1}\right) \stackrel{\text { def }}{=} X_{1}^{\prime}=X_{1}+T \wedge X_{3}^{\prime}=X_{3}\left(v_{2}\right) \stackrel{\text { def }}{=} X_{2}^{\prime}=X_{2}+T \\
D y n_{1}\left(v_{1}^{\prime}\right) \stackrel{\text { def }}{=} X_{1}^{\prime}=0 \wedge X_{3}^{\prime}=X_{3}+T & \\
\operatorname{Res}_{1}\left(e_{1}\right) \stackrel{\text { def }}{=} X_{1}^{\prime}=0 \wedge X_{3}^{\prime}=0 & \operatorname{Res}_{2}\left(e_{2}\right) \stackrel{\text { def }}{=} X_{2}^{\prime}=0 \\
\operatorname{Res}_{1}\left(e_{1}^{\prime}\right) \stackrel{\text { def }}{=} X_{1}^{\prime}=0 \wedge X_{3}^{\prime}=0 \wedge X_{2}^{\prime}=0 & \\
\operatorname{Inv}_{1}\left(v_{1}\right) \stackrel{\text { def }}{=} X_{1} \leq 1, \operatorname{Inv}_{1}\left(v_{1}^{\prime}\right) \stackrel{\text { def }}{=} X_{1}=0 & \operatorname{Inv}_{2}\left(v_{2}\right) \stackrel{\text { def }}{=} X_{2} \leq \sqrt{2} \\
\operatorname{Act}_{1}\left(e_{1}\right) \stackrel{\text { def }}{=} X_{1}=1, A c t_{1}\left(e_{1}^{\prime}\right) \stackrel{\text { def }}{=} X_{1}+X_{2}>2 & \operatorname{Act}_{2}\left(e_{2}\right) \stackrel{\text { def }}{=} X_{2}=\sqrt{2}
\end{array}
$$
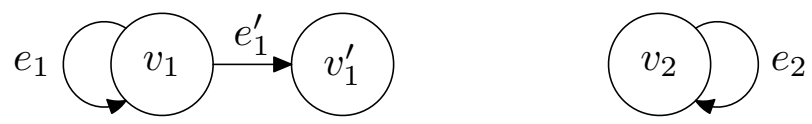

Fig. 4. The discrete projection of $H_{1}$ and $H_{2}$ 
Since $H_{1}$ and $H_{2}$ share the variable $X_{2}$, we cannot model their synchronous evolution by using parallel composition. However, one may notice that all the formulæ involving $X_{2}$ in $H_{1}$ are related with $e_{1}^{\prime}$. Let $H_{1}^{\prime}$ be the automaton obtained by removing $e_{1}^{\prime}$ from $H_{1}$. It is easy to see that the concurrent evolution of $H_{1}$ and $\mathrm{H}_{2}$ is representable by the hybrid automaton $H_{*}$ obtained by providing $H_{1}^{\prime} \otimes H_{2}$ of a further edge $\bar{e}$, from $\left\langle v_{1}, v_{2}\right\rangle$ to $\left\langle v_{1}^{\prime}, v_{2}\right\rangle$, whose activation and reset formulæ are $\operatorname{Act}_{1}\left(e_{1}^{\prime}\right)$ and $\operatorname{Res}_{1}\left(e_{1}^{\prime}\right)$, respectively.

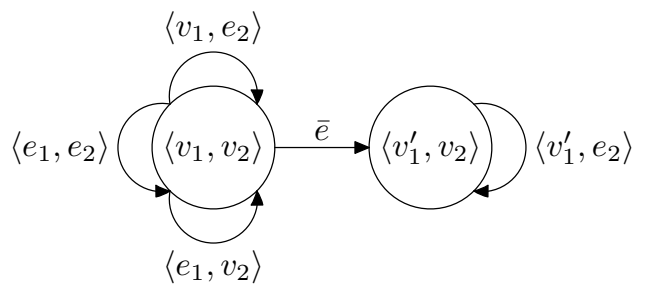

Fig. 5. The discrete projection of $H_{*}$

It follows that $F$ is reachable from $I$ in $H_{*}$ if and only if either $F$ is reachable from $I$ or $A c t_{1}\left(e_{1}^{\prime}\right)$ is reachable from $I$ and $F$ is reachable from $\operatorname{Res}_{1}\left(e_{1}^{\prime}\right)$ in $H_{1}^{\prime} \otimes H_{2}$. Hence, we can exploit the results presented in this paper to decide reachability property on $H_{*}$. Notice that a similar approach can be used also when shared variables appear in either dynamics or invariants. We leave both formal definition and applicability analysis of synchronous composition for future work.

\section{Conclusions}

This paper extends our earlier work [1] showing that the reachability problem for parallel composition of semi-algebraic o-minimal hybrid automata is decidable. To achieve such a result, it exploited Tarski's decidability result on semi-algebraic theory, density results over $\mathbb{R}$, algorithms for the membership problems over algebraic fields, and algorithms for solving systems of linear Diophantine systems. Further, by showing that this class of automata does not admit a finite simulation quotient (see [1,2]), we have proved impossibility of obtaining such a result through standard finite quotient techniques.

Time-complexity issues limit the practical applicability of our result. Nevertheless, it presents some intriguing theoretical features. Note first that, to prove the decidability of parallel composition, we took advantage of the decidability of a rather simple mixed real/integer problem. Such mixed approaches, in some sense, reflect the continuous-discrete behavior described by hybrid systems.

Our decidability results may be surprising, in the context of Miller's undecidability results [11], but can be explained as follows. While we require constant resets on automata components, Miller admitted both constant and identity resets. Hence, he could test the value of a variable through an activation and do 
not change it by applying an identity reset. This is a fundamental gadget in the construction of the two-counter Minsky machine encoding needed to prove the undecidability result (see Figure 3 in [11]), but it is inapplicable in our case.

Finally, the technique of this paper emphasizes the hardest cases to decide and suggests a class of automata for which the reachability problem of parallel composition can be reduced to reachability problems on each component.

\section{References}

1. Casagrande, A., Corvaja, P., Piazza, C., Mishra, B.: Composing semi-algebraic o-minimal automata. In: Proc. of Hybrid Systems: Computation and Control (HSCC’07). LNCS, Springer (2007) 668-671 Short paper.

2. Casagrande, A., Corvaja, P., Piazza, C., Mishra, B.: Parallel composition of semi-algebraic o-minimal automata. Available at http://www.dimi.uniud.it/piazza/PAPERS/parallel.pdf (January 2008)

3. Alur, R., Henzinger, T.A., Ho, P.H.: Automatic Symbolic Verification of Embedded Systems. In: IEEE Real-Time Systems Symposium '93, IEEE Press (1993) 2-11

4. Henzinger, T.A., Kopke, P.W., Puri, A., Varaiya, P.: What's decidable about hybrid automata? In: Proc. of Symp. on Theory of Computing (STOCS'95). (1995) 373-382

5. Lafferriere, G., Pappas, G.J., Sastry, S.: O-minimal Hybrid Systems. Mathematics of Control, Signals, and Systems 13 (2000) 1-21

6. Brihaye, T., Michaux, C., Rivière, C., Troestler, C.: On O-Minimal Hybrid Systems. In: Proc. of Hybrid Systems: Computation and Control (HSCC'04). Volume 2993 of LNCS., Springer (2004) 219-233

7. Tarski, A.: A Decision Method for Elementary Algebra and Geometry. Univ. California Press (1951)

8. van den Dries, L.: Tame Topology and O-minimal Structures. Volume 248 of London Mathematical Society Lecture Note Series. Cambridge University Press (1998)

9. Casagrande, A., Piazza, C., Mishra, B.: Semi-Algebraic Constant Reset Hybrid Automata - SACoRe. In: Proc. of the 44rd Conference on Decision and Control (CDC'05), IEEE Press (2005) 678-683

10. Henzinger, T.A.: The Theory of Hybrid Automata. In: Proc. of IEEE Symposium on Logic in Computer Science (LICS'96), IEEE Press (1996) 278-292

11. Miller, J.S.: Decidability and Complexity Results for Timed Automata and Semilinear Hybrid Automata. In: Proc. of Hybrid Systems: Computation and Control (HSCC'00). Volume 1790 of LNCS., Springer (2000) 296-309

12. Pottier, L.: Minimal solutions of linear diophantine systems: Bounds and algorithms. In Book, R.V., ed.: Proc. 4th Conference on Rewriting Techniques and Applications. Volume 488., Springer (1991) 162-173

13. Bourbaki, N.: Elements of Mathematics. General topology II. Springer (1989)

14. Cohen, H.: A Course in Computational Algebraic Number Theory. Volume 138 of Graduate Texts in Mathematics. Springer (1993)

15. Casagrande, A., Corvaja, P., Piazza, C., Mishra, B.: Synchronized product of semialgebraic o-minimal hybrid automata. Technical report, University of Udine (October 2006) Available at http://fsv.dimi.uniud.it/papers/syncro.pdf.

16. Casagrande, A., Casey, K., Falchi, R., Piazza, C., Ruperti, B., Vizzotto, G., Mishra, B.: Translating Time-Course Gene Expression Profiles into Semi-algebraic Hybrid Automata Via Dimensionality Reduction. In: Proc. of Conference on Algebraic Biology (AB'07). Volume 4545 of LNCS., Springer (2007) 51-65 